\title{
The Outage Capacity of Linear Space-Time Codes
}

\author{
Badri Varadarajan and John R. Barry, Senior Member, IEEE
}

\begin{abstract}
An inner space-time code, i.e., one that is complemented by an outer error-control code, calls for vastly different design strategies than a space-time code that stands alone. This letter investigates the design of a linear inner space-time code for a $t$-input $\boldsymbol{r}$-output Rayleigh fading channel by examining its outage capacity, which assumes an idealized outer code. We show that a linear space-time code with rate $R<\min (t, r)$ can achieve at most a fraction $R / \min (t, r)$ of the underlying channel's outage capacity at high signal-to-noise ratio (SNR). Conversely, we find that a space-time code with low raw diversity order (as calculated using the rank rule) does not necessarily suffer a capacity penalty. Under very general conditions, a rate of $R=\min (t, r)$ is sufficient to ensure that the outage capacity of the space-time code approaches that of the underlying channel at high SNR. Simulation results are presented to support the claims.
\end{abstract}

Index Terms-Diversity order, multiple-input multiple-output (MIMO), multiplexing-diversity tradeoff, multiplexing gain.

\section{INTRODUCTION}

$\mathbf{T}$ HE PROMISE of dramatic diversity and multiplexing gains [1] has led to the widespread adoption of multiple transmit and receive antennas in wireless communications systems. To realize these potential gains, special coding techniques are required. One way to harness the diversity gain is to use space-time codes [2] at the transmitter front end. Early space-time codes [3], [4] were designed assuming that their inputs are drawn from a discrete alphabet independently for every space-time code block, without an outer error-control code. The diversity gain of such a stand-alone space-time code is called its raw diversity order, and can be calculated using the rank rule [2]. The multiplexing gain of a stand-alone space-time code is quantified by its rate, defined as the average number of information symbols transmitted in one signaling interval across the multiple-input multiple-output (MIMO) fading channel. Stand-alone space-time codes with maximum rate and maximum raw diversity order have been designed (for example [5]).

In many practical communication systems [6]-[8], the space-time code does not stand alone, but is concatenated with an outer error-correction code. The presence of the outer code changes the role of the space-time code, calling for new design approaches [9]. One popular approach [10] is to treat the combination of the space-time inner code and the MIMO fading channel as an effective channel. Then, the actual outer code, which operates across the effective channel, is replaced

Manuscript received September 26, 2003; revised September 9, 2004; accepted December 29,2004. The editor coordinating the review of this paper and approving it for publication is A. Conti. This work was supported by the National Science Foundation under Grants CCR-0082329 and CCR-0121565.

The authors are with the School of Electrical and Computer Engineering, Georgia Institute of Technology, Atlanta, GA 30332 USA (e-mail: badri@ece. gatech.edu; barry@ece.gatech.edu).

Digital Object Identifier 10.1109/TWC.2005.858329 by an idealized infinite-length code with a Gaussian alphabet. Idealizing the outer code helps to maintain focus on the inner space-time code alone. Now, information-theoretic analysis of the effective channel accurately yields the data rates and error rates that can be supported by the idealized concatenated transmitter, and hence, furnishes performance metrics to evaluate the goodness of space-time inner codes. In this paper, we focus on the outage capacity [8], [11] of the effective channel for linear space-time codes, also known as linear dispersion codes [9], [10]. Specifically, we study the impact of rate and raw diversity order of space-time codes on their outage capacity.

The impact of a linear space-time code's rate on its outage capacity has been analyzed extensively in the literature. In [10] and [12], it was shown that, when operating over a $t$-input $r$-output Rayleigh fading channel, a linear space-time code with rate less than $\min (t, r)$ suffers a nonzero loss in outage capacity when compared to the underlying MIMO fading channel itself. However, the capacity loss was not quantified. More precise results are available for orthogonal block codes [4] such as the Alamouti code [3]. An orthogonal block code with rate $R<1$ operating over a $t$-input 1-output Rayleigh fading channel achieves only a fraction $R$ of the fading channel's outage capacity at any signal-to-noise ratio (SNR) [13], [14]. More generally, the multiplexing-diversity tradeoff curve for orthogonal block codes [1] shows that, at high SNR, a rate$R$ orthogonal design achieves a fraction $R / \min (t, r)$ of the outage capacity of a $t$-input $r$-output Rayleigh fading channel. In this paper, we generalize this result and show that any rate- $R$ linear space-time code achieves at most a fraction $R / \min (t, r)$ of the Rayleigh fading channel's outage capacity at high SNR.

This paper is organized as follows. In Section II, we define and discuss the diversity and multiplexing orders of Rayleighfading MIMO channels. In Sections III and IV, we analyze, respectively, the diversity and multiplexing orders achieved by a linear space-time inner code, when a capacity-achieving outer code is present. In Section V, we discuss an example that illustrates the effect of multiplexing order and achievable diversity order on the outage capacity of linear space-time codes. In Section VI, we interpret our analysis and present simulation results to support our interpretation. Section VII summarizes our conclusions.

\section{Diversity AND MultipleXing ORDER OF MIMO CHANNELS}

We consider a $t$-input $r$-output linear quasi-static narrowband fading channel, modeled as

$$
\mathbf{y}_{k}=\mathbf{H x}_{k}+\mathbf{n}_{k}
$$

where $\mathbf{x}_{k}$ is the $t \times 1$ channel input and $\mathbf{y}_{k}$ the $r \times 1$ channel output at time $k$. The noise $\mathbf{n}_{k}$ is spatially and temporally white, 
so that $\mathbf{E}\left[\mathbf{n}_{k+l} \mathbf{n}_{k}^{*}\right]=\delta_{l} N_{0} \mathbf{I}_{r}$. We assume that the random $r \times t$ channel matrix $\mathbf{H}$ is unknown to the transmitter, but known to the receiver. Further, we make the Rayleigh fading assumption, wherein the entries of $\mathbf{H}$ are independent circularly symmetric Gaussian random variables with zero mean and variance one. The SNR $S$ is defined as the ratio of the average received signal energy to the average received noise energy, namely $S=E\left[\left\|\mathbf{H x}_{k}\right\|^{2}\right] / r N_{0}$.

Suppose a communication system operates at a fixed data rate $R_{\mathrm{b}}$ bits per signaling interval across the MIMO fading channel (1). Error-free communication can be guaranteed if and only if $R_{\mathrm{b}}$ is less than or equal to the available capacity of the random MIMO channel. Constrained by the transmitter's ignorance of the random channel matrix $\mathbf{H}$, the available capacity is given by [11]

$$
I(S, \mathbf{H})=\log \operatorname{det}\left(\mathbf{I}_{r}+\frac{S}{t} \mathbf{H H}^{*}\right) .
$$

The event that $I(S, \mathbf{H})$ is less than $R_{\mathrm{b}}$ is called an outage. Clearly, the outage probability, given by

$$
F\left(S, R_{\mathrm{b}}\right)=\operatorname{Pr}\left[I(S, \mathbf{H})<R_{\mathrm{b}}\right]
$$

depends on both the data rate $R_{\mathrm{b}}$ and the SNR $S$. For a fixed data rate, the outage probability decreases as the SNR increases. The rate of this decrease at asymptotically high SNR is measured by the diversity order, namely

$$
\delta=-\lim _{S \rightarrow \infty} \frac{\log F\left(S, R_{\mathrm{b}}\right)}{\log S} .
$$

Graphically, the diversity order is the asymptotic slope of outage probability versus SNR plotted on a $\log -\log$ scale. The function $F\left(S, R_{\mathrm{b}}\right)$ is known for Rayleigh fading channels [11], and can be used to compute the limit (4) to obtain the following result [1].

Theorem 1: The diversity order of a $t$-input $r$-output Rayleigh fading channel is $t r$.

Instead of fixing the data rate, one can fix the tolerable error probability and design the communication system to achieve the maximum possible data rate. Given the SNR $S$ and the maximum outage probability $p_{\mathrm{o}}$, the maximum possible data rate is called the outage capacity, namely

$$
C\left(S, p_{\mathrm{o}}\right)=\sup \left\{R_{\mathrm{b}}: F\left(S, R_{\mathrm{b}}\right)<p_{\mathrm{o}}\right\} .
$$

Analogous to diversity order, we define the multiplexing order $\mu$ as the asymptotic slope of an outage capacity versus log SNR plot, namely

$$
\mu=\lim _{S \rightarrow \infty} \frac{C\left(S, p_{\mathrm{o}}\right)}{\log S} .
$$

The following result is well known in the theory of Rayleigh fading channels [1], [8], [11]. ${ }^{1}$

\footnotetext{
${ }^{1}$ Theorem 2 also holds for a wide class of non-Rayleigh fading channels; it only requires that the channel matrix $\mathbf{H}$ be full rank with probability 1 . This follows as a corollary of Theorem 3, which we state and prove in Section IV.
}

Theorem 2: The multiplexing order of a $t$-input $r$-output Rayleigh fading channel is $\min (t, r)$.

One way to harvest the diversity gain of MIMO fading channels is to use linear space-time codes (or linear dispersion codes) [10] at the transmitter front end. In the next two sections, we discuss the diversity and multiplexing orders of a linear space-time inner code, when a capacity-achieving outer code is present.

\section{ACHIEVABLE DIVERSITY ORDER OF SPACE-TIME CODES}

A space-time code takes in a $K \times 1$ vector $\mathbf{u}$ of complex input symbols, and produces $t \times 1$ channel-input vectors $\mathbf{x}_{1}, \mathbf{x}_{2}, \ldots, \mathbf{x}_{N}$ for $N$ signaling intervals. Such a space-time code is said to have length $N$ and rate $R=K / N$. Define the $N t \times 1$ composite transmit vector as $\mathbf{x}=\left[\mathbf{x}_{1}^{\mathrm{T}}, \mathbf{x}_{2}^{\mathrm{T}}, \ldots, \mathbf{x}_{N}^{\mathrm{T}}\right]^{\mathrm{T}}$. For a linear space-time code, each output symbol in $\mathrm{x}$ is a linear combination of the $K$ input symbols in $\mathbf{u}$ and their complex conjugates. To compactly represent the encoding process, we use the complex-to-real transformations [11]

$$
\hat{\mathbf{b}}=\left[\begin{array}{c}
\operatorname{Re}(\mathbf{b}) \\
\operatorname{Im}(\mathbf{b})
\end{array}\right] \quad \text { and } \quad \hat{\mathbf{A}}=\left[\begin{array}{cc}
\operatorname{Re}(\mathbf{A}) & -\operatorname{Im}(\mathbf{A}) \\
\operatorname{Im}(\mathbf{A}) & \operatorname{Re}(\mathbf{A})
\end{array}\right]
$$

for complex vectors $\mathbf{b}$ and matrices $\mathbf{A}$. By definition, a space-time code is said to be linear when the composite transmit vector $\mathbf{u}$ is related to the input vector $\mathbf{u}$ by [12]

$$
\hat{\mathbf{x}}=\mathbf{M} \hat{\mathbf{u}} .
$$

The $2 N t \times 2 K$ real matrix $\mathbf{M}$ is called the encoding matrix and completely specifies the linear space-time code. Once $\hat{\mathbf{x}}$ is obtained using (8), its elements are rearranged to yield the $N$ transmit vectors $\mathbf{x}_{1}, \mathbf{x}_{2}, \ldots, \mathbf{x}_{N}$, which are then transmitted across the MIMO channel (1). The composite receive vector per block is defined as $\mathbf{y}=\left[\mathbf{y}_{1}^{\mathrm{T}}, \mathbf{y}_{2}^{\mathrm{T}}, \ldots, \mathbf{y}_{N}^{\mathrm{T}}\right]^{\mathrm{T}}$. Clearly

$$
\mathbf{y}=\left[\begin{array}{cccc}
\mathbf{H} & \mathbf{0} & \ldots & \mathbf{0} \\
\mathbf{0} & \mathbf{H} & \ddots & \vdots \\
\vdots & \ddots & \ddots & \mathbf{0} \\
\mathbf{0} & \ldots & 0 & \mathbf{H}
\end{array}\right] \mathbf{x}+\mathbf{n}
$$

where $\mathbf{n}=\left[\mathbf{n}_{1}^{\mathrm{T}}, \mathbf{n}_{2}^{\mathrm{T}}, \ldots, \mathbf{n}_{n}^{\mathrm{T}}\right]^{\mathrm{T}}$. Letting $\mathbf{G}$ denote the block diagonal matrix in (9), the transformations (7) yield $\hat{\mathbf{y}}=$ $\hat{\mathbf{G x}}+\hat{\mathbf{n}}$. Further, substituting $\hat{\mathbf{x}}=\mathbf{M} \hat{\mathbf{u}}$ from (8) gives

$$
\hat{\mathbf{y}}=\hat{\mathbf{G M}} \mathbf{\mathbf { u }}+\hat{\mathbf{n}} \text {. }
$$

Equation (10) is the input-output relation of the effective channel formed by the combination of the linear space-time code and the Rayleigh fading channel (1). Note that the effective channel is a linear MIMO channel, whose transfer matrix $\mathbf{H}_{\text {eff }}=\hat{\mathbf{G M}}$ is real and has dimension $2 N r \times 2 K$. Further, $\mathbf{H}_{\text {eff }}$ depends on the random fading channel matrix $\mathbf{H}$. For any value of $\mathbf{H}$, it is easy to show [12] that the available capacity of 
the effective channel, namely, the highest supportable data rate with an infinite-length Gaussian-alphabet outer code, is

$$
J(S, \mathbf{H})=\log \operatorname{det}\left(\mathbf{I}_{2 N r}+\alpha \frac{S}{t} \mathbf{H}_{\mathrm{eff}} \mathbf{H}_{\mathrm{eff}}^{\mathrm{T}}\right)
$$

where $\alpha=2 N t \operatorname{tr}\left(\mathbf{M}^{\mathrm{T}} \mathbf{M}\right)$. For a data rate $R_{\mathrm{b}}$, the outage probability of the effective channel is

$$
G\left(S, R_{\mathrm{b}}\right)=\operatorname{Pr}\left[J(S, \mathbf{H})<R_{\mathrm{b}}\right]
$$

Analogous to (4), the diversity order of the effective channel is defined as

$$
\delta_{\mathrm{eff}}=-\lim _{S \rightarrow \infty} \frac{\log G\left(S, R_{\mathrm{b}}\right)}{\log S}
$$

Note that $\delta_{\text {eff }}$ is the diversity gain achieved when an infinitelength outer code with a Gaussian output alphabet is concatenated with the given space-time inner code. Since an outer code is required to achieve the diversity gain $\delta_{\text {eff }}$, we call it the achievable diversity order of the space-time code. In contrast, the raw diversity order of the space-time code is the diversity gain obtained by the space-time code alone, with uncoded inputs drawn from a discrete alphabet, independently from one space-time code block to the next. Given the input alphabet, the raw diversity order is obtained using the rank rule [2]. On the other hand, it is an open problem to compute the limit (13), and obtain the achievable diversity order of a space-time code.

One important observation is that space-time codes with low raw diversity order can potentially have high achievable diversity order. As an example, consider the serialto-parallel ( $\mathrm{S} / \mathrm{P})$ converter that is used as space-time code in the Vertical Bell Laboratories Layered Space-Time Architecture (V-BLAST) transmitter [15]. It has length $N=1$, rate $R=t$, and its encoding rule is simply $\mathbf{x}=\mathbf{u}$. From the rank rule, it is easy to show that the raw diversity order of the $\mathrm{S} / \mathrm{P}$ converter is $r \min (t, N)=r$, since $N=1$. On the other hand, since $\mathbf{x}=\mathbf{u}$, the S/P converter really does no space-time coding. Therefore, the effective channel is the same as the original channel, and the achievable diversity order is equal to the diversity order (4) of the original MIMO fading channel, namely $t r$.

The example of the $\mathrm{S} / \mathrm{P}$ converter indicates that the raw diversity order of a space-time code does not limit its diversity gain in the presence of an outer code. On the other hand, we will show in the next section that the rate of a space-time code limits its multiplexing gain, even with a powerful outer code.

\section{Achievable Multiplexing ORDER OF SPACE-TIME CODES}

Analogous to (5) for the MIMO fading channel, the outage capacity of the effective channel is defined as

$$
D\left(S, p_{\mathrm{o}}\right)=\sup \left\{R_{\mathrm{b}}: G\left(S, R_{\mathrm{b}}\right)<p_{\mathrm{o}}\right\}
$$

Extending the analogy, the multiplexing order of the effective channel is

$$
\mu_{\mathrm{eff}}=\lim _{S \rightarrow \infty} \frac{D\left(S, p_{\mathrm{o}}\right)}{\log S} .
$$

In the remainder of this section, we analyze $\mu_{\text {eff }}$. The following result gives an upper bound on $\mu_{\text {eff }}$, and also gives a condition under which the upper bound is reached.

Theorem 3: The multiplexing order $\mu_{\mathrm{eff}}$ of a rate- $R$ linear space-time code operating over a $t$-input $r$-output Rayleigh fading channel satisfies

$$
\mu_{\mathrm{eff}} \leq n=\min (t, r)
$$

Furthermore, equality is reached $\left(\mu_{\mathrm{eff}}=n\right)$ whenever the effective channel matrix $\mathbf{H}_{\text {eff }}=\hat{\mathbf{G M}}$ has full rank with probability 1 .

Theorem 3 is proved in Appendix I. The upper bound (16) implies that the multiplexing order of a linear space-time code is limited by its rate. To achieve the upper bound, the encoding matrix $\mathbf{M}$ should be chosen to ensure that $\hat{\mathbf{G} M}$ has full rank with probability 1 . Most practical space-time codes do have encoding matrices satisfying this property, as seen from the following result.

Theorem 4: A linear space-time code with rate $R \leq$ $\min (t, r)$ operating over a $t$-input $r$-output Rayleigh fading channel achieves a multiplexing order $\mu_{\mathrm{eff}}=\min (t, r, R)=R$ only if its encoding matrix $\mathbf{M}$ has full rank. Further, we have the following conditions.

1) If $r \geq t$, any full rank $\mathbf{M}$ is sufficient to achieve $\mu_{\mathrm{eff}}=R$.

2) If $r<t, \mathbf{M}$ having full rank does not suffice to achieve $\mu_{\text {eff }}=R$. However, it is sufficient (though not necessary) to ensure that for all $i=1,2, \ldots, N$, the transmit vector $\mathbf{x}_{i}$ always belongs to some complex linear space $\mathcal{S}_{i}$, whose dimension $l_{i}$ satisfies $1 \leq l_{i} \leq r$.

Theorem 4 is proved in Appendix II. A practical linear space-time code is likely to have a full-rank encoding matrix $\mathbf{M}$ anyway, in order to ensure that the encoding process $\hat{\mathbf{x}}=\mathbf{M} \hat{\mathbf{u}}$ is easily reversible. Thus, from condition 1) of Theorem 4, every practical linear space-time code achieves $\mu_{\mathrm{eff}}=R$ when $r \geq t$.

When $r<t$, a full-rank encoding matrix is necessary but not sufficient to ensure $\mu_{\mathrm{eff}}=R$. To see the intuition behind the sufficient condition 2, note that the dimension of the set $\mathcal{X}_{i}$ containing all possible $\mathbf{x}_{i}$ represents the number of symbols multiplexed by the $i$ th channel-input vector. Condition 2 says that in order to achieve $\mu_{\mathrm{eff}}=R$, it is sufficient to ensure that the number of symbols multiplexed in each signaling interval is less than or equal to the multiplexing ability of the MIMO channel, namely $\min (t, r)=r$. Note that the average number of symbols multiplexed per signaling interval, namely the rate $R$, is assumed to be less than or equal to $r$. Thus, condition 2 equivalently requires that the average multiplexing rate of the space-time code should be distributed roughly uniformly among all the signaling intervals in one block.

To illustrate the sufficient condition 2 , consider two linear space-time codes with length $N=2$ and rate $R=1$ operating 
over a Rayleigh fading channel with $t=2$ inputs and $r=1$ output. For this combination, the upper bound on multiplexing order is $n=\min (t, r, R)=1$. Also, there are $K=N R=2$ symbols, say $u_{1}$ and $u_{2}$, in the input vector $\mathbf{u}$. Consider first a space-time code with transmit vectors $\mathbf{x}_{1}=\mathbf{u}$ and $\mathbf{x}_{2}=\mathbf{0}$ in one code block. This space-time code uses the 2-input 1-output Rayleigh fading channel every alternate time instant, achieving exactly half the channel's outage capacity and hence, half its multiplexing order of $\min (t, r)=1$. Thus, the actual multiplexing order of the code is $1 / 2$, which is less than the upper bound $\min (t, r, R)=1$. This is not surprising since the sufficient condition 2 of Theorem 4 is violated: the two transmit vectors $\mathbf{x}_{1}$ and $\mathbf{x}_{2}$ belong to sets of dimension 2 and 0 , respectively, instead of $r=1$ each, as required by condition 2 . On the other hand, consider the linear code whose transmit vectors are $\mathbf{x}_{1}=\left[\begin{array}{ll}u_{1} & 0\end{array}\right]^{\mathrm{T}}$ and $\mathbf{x}_{2}=\left[\begin{array}{ll}u_{2} & 0\end{array}\right]^{\mathrm{T}}$. This code uses only the first transmit antenna, and always transmits 0 out of the second antenna. Thus, the effective channel for this code is a 1-input 1-output Rayleigh fading channel with multiplexing order 1 , meeting the upper bound $n=1$. This is to be expected, since this code satisfies the sufficient condition 2 of Theorem 4: both transmit vectors have dimension 1, as required.

We remark that condition 2 is only a sufficient condition for a space-time code to achieve $\mu_{\mathrm{eff}}=R$, but not a necessary condition. For example, the length-two rate-one Alamouti code operating over a 2-input 1-output Rayleigh fading channel does not satisfy the condition, since both transmit vectors belong to spaces of dimension 2. However, it is easy to show that the effective channel matrix for the Alamouti code has full rank with probability 1 . Hence, from Theorem 3, the Alamouti code does achieve a multiplexing order of $n=\min (2,1,1)=1$.

\section{Outage Capacity Asymptote}

The multiplexing order is, by definition, the slope of the outage capacity versus log SNR asymptote. From Theorem 2, a $t$-input $r$-output Rayleigh fading channel has an asymptote of slope $\min (t, r)$. On the other hand, Theorems 3 and 4 say that a linear space-time code with rate $R<\min (t, r)$ operating over the same Rayleigh fading channel can be designed to achieve a multiplexing order of $R$, but no more. Thus, a lowrate space-time code has a shallower outage capacity asymptote than the channel on which it operates. On the other hand, the effect of low achievable (not raw) diversity order is to reduce the offset of the space-time code's capacity asymptote, as shown in [16] and [17].

We now discuss an example to illustrate the effect of rate and achievable diversity order of a space-time code on its outage capacity. We compare two rate-one space-time codes operating over a 2-input $r$-output Rayleigh fading channel. The first code is the Alamouti code [3], which is known to have full achievable diversity order $2 r$. The second code is the repetition code, which takes in one complex symbol every signaling interval and simultaneously transmits it from both transmit antennas. It is easy to show that the effective channel for the repetition code is equivalent to a 1-input $r$-output Rayleigh

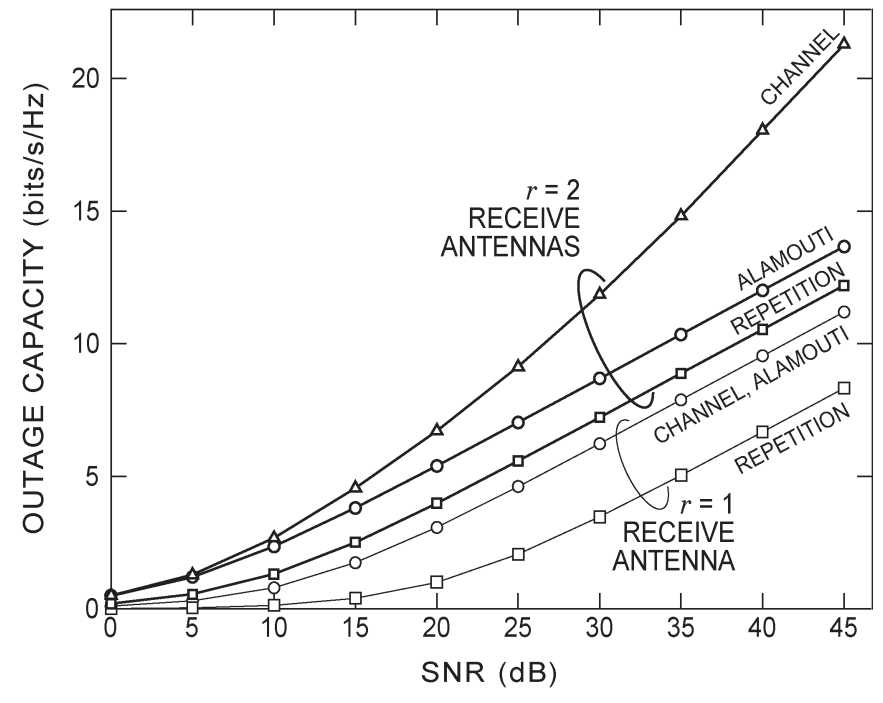

Fig. 1. Outage capacity versus SNR at $1 \%$ outage, assuming $t=2$ transmit antennas.

fading channel. Hence, the repetition code has an achievable diversity order of only $r$. We will consider two different numbers of channel outputs, namely $r=1$ and $r=2$. For both cases, the $1 \%$ outage capacity of the Rayleigh fading channel and for the two space-time codes is plotted against SNR in Fig. 1.

Consider first the case of $r=1$ channel output. The 2-input 1-output Rayleigh fading channel has multiplexing order $\min (2,1)=1$. From Theorem 3, the Alamouti and repetition codes also have multiplexing order $n=\min (2,1,1)=1$ (the upper bound $n$ is reached because the effective channel matrices can easily be shown to have full rank with probability 1 ). This is verified by Fig. 1, where the asymptotic slopes of the Alamouti and repetition-code capacity curves match that of the 2-input 1-output channel. In addition to full multiplexing order, the Alamouti code also has full achievable diversity order $t r=2$, so we expect it to lose very little capacity. Remarkably, as observed in Fig. 1 and proven in [14], the capacity penalty of the Alamouti code is 0 when there is only one receive antenna. The repetition code, on the other hand, has achievable diversity order of just $r=1$. The low achievable diversity order results in an asymptotic offset loss (or capacity loss that saturates at high SNR), as seen in Fig. 1.

With $r=2$ outputs, the diversity order of the 2-input 2-output Rayleigh fading channel is $t r=4$, and its multiplexing order is $\min (t, r)=2$. On the other hand, from Theorem 3 , the multiplexing orders of the Alamouti and repetition codes still equal $\min (2,2,1)=1$. This agrees with Fig. 1, where the outage capacity curve of the 2-input 2-output Rayleigh fading channel is twice as steep as the capacity curves for the two space-time codes. The Alamouti code at least has full achievable diversity order of 4 . On the other hand, the repetition code has achievable diversity order of only $r=2$, hence it suffers an additional offset loss when compared to the Alamouti code.

We emphasize that the repetition code is a pathological example. Typically, space-time codes have full achievable 
diversity order of $t r$, as illustrated by the example of the S/P converter in Section III.

\section{IMPORTANCE OF HIGH RATE FOR SPACE-TIME INNER CODES}

From the multiplexing order analysis, it is clear that a space-time inner code with rate $R$ less than $\min (t, r)$ achieves at most a fraction $R \leq \min (t, r)$ of the achievable data rate in the presence of a powerful outer code. To avoid this loss, space-time inner codes should have a rate of at least $\min (t, r)$ and must be designed in keeping with the conditions of Theorem 4. On the other hand, even a space-time code with low raw diversity order has high achievable diversity order in the presence of an outer code. Combining the two observations, we conclude that to harvest the multiplexing and diversity gains of MIMO fading channels, space-time inner codes should have full rate and full achievable diversity order, but not necessarily full raw diversity order. Strictly, this conclusion is based on information-theoretic analysis, which assumes that the outer code has infinite length and a Gaussian output alphabet. However, we conjecture that the conclusion holds even for powerful binary error-control codes like turbo and low-density parity check codes. To support this conjecture, we present simulation results in this section.

We compare two space-time inner codes operating over a 4-input 4-output Rayleigh-fading channel, when the outer code is a rate- $2 / 3(4800,3200)$ turbo code. The turbo code is a parallel concatenation of a pair of $\left[1,\left(1+D+D^{4}\right) /(1+\right.$ $\left.\left.D+D^{2}+D^{3}+D^{4}\right)\right]$ convolutional encoders, separated by a spread-20 interleaver, with puncturing so that only the parity bits $4 k$ and $4 k+2$ are transmitted from the two encoders, respectively. Following the bit-interleaved coded modulation strategy [6], the 4800 turbo-coded bits are interleaved using a spread-24 interleaver before being Gray mapped to complex quadratic-amplitude modulation (QAM) symbols for space-time encoding. The two space-time codes considered are the $\mathrm{S} / \mathrm{P}$ converter and a general layered space-time (GLST) code [18], which consists of two parallel Alamouti codes, each operating over a different set of two channel inputs. The S/P converter has rate 4 , and raw diversity order equal to the number of channel outputs, namely 4. It uses 16-QAM modulation, yielding a total data rate of $10.67 \mathrm{bits} / \mathrm{s} / \mathrm{Hz}$. On the other hand, the GLST code has rate 2 and consequently has to use 256-QAM modulation to achieve the same data rate. The GLST code has higher raw diversity order than the S/P converter, namely 8 [18]. The receiver performs iterative decoding between the outer turbo decoder and a soft-output list sphere decoder for the inner space-time code [6]. Three turbo iterations are performed for each of the ten iterations between the error-control decoder and the space-time detector. Fig. 2 shows a plot of the worderror rate versus SNR. Each point on the curve represents an observation of at least 150 word errors.

The multiplexing order of the $\mathrm{S} / \mathrm{P}$ converter is equal to that of the channel itself, namely $\min (4,4)=4$. In contrast, due to its low rate, the multiplexing order of the GLST code is $\min (4,4,2)=2$ from Theorem 4 (since the number of transmit

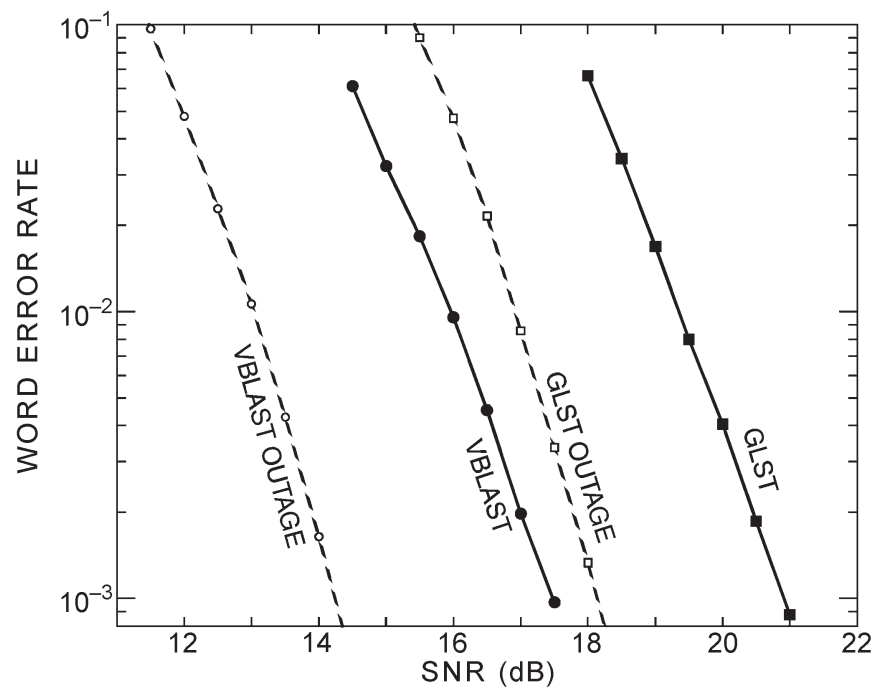

Fig. 2. Comparing the S/P converter and GLST space-time codes for a $4 \times 4$ channel with a turbo outer code, at $10.67 \mathrm{bits} / \mathrm{s} / \mathrm{Hz}$.

and receive antennas is equal, the full-rank encoding matrix of the GLST code is sufficient to guarantee this). We expect that the low multiplexing order of the latter code should lead to a loss of capacity, and hence, poor performance in the presence of the outer turbo code. This is confirmed by the plot, which shows the high-rate S/P converter outperforming the low-rate GLST encoder by nearly $3.5 \mathrm{~dB}$. For comparison, the outage probability is plotted against SNR, with the data rate fixed at $10.67 \mathrm{bits} / \mathrm{s} / \mathrm{Hz}$. Note that even with a codelength of just 4800 bits, the performance of the turbo code is within $3 \mathrm{~dB}$ of the outage-probability curve at a word-error rate of $10^{-3}$. Thus, the turbo code does get higher diversity gain than the raw diversity order of the S/P converter, as expected. However, from the shallower slopes of the performance curves when compared to the outage-probability curves, it is clear that the turbo code is not strong enough to achieve the full diversity order of the channel. We conjecture that increasing the length of the turbo code will achieve full diversity order.

\section{CONCLUSION}

We studied the design of space-time inner codes for concatenated transmitter architectures. The raw diversity order of a space-time code, as obtained by the rank rule, is only a lower bound to the achievable diversity order in the presence of a powerful outer code. Therefore, the raw diversity order of the space-time inner code does not limit diversity gain of the concatenated transmitter. On the other hand, we showed that the rate of the space-time inner code does limit the multiplexing gain of the concatenated transmitter. More precisely, we showed that a space-time code with rate $R<\min (t, r)$ achieves at most a fraction $R / \min (t, r)$ of the channel's outage capacity at high SNR. To actually achieve the above upper bound, it is necessary to use a full-rank encoding matrix. When $t \leq r$, any full-rate encoding matrix achieves the upper bound. For the case $t>r$, we gave a sufficient condition for achieving the upper bound. 
We conclude that, in order to approach the channel's outage capacity at high SNR, a space-time inner code should have a rate of at least $\min (t, r)$, but need not have high raw diversity order. This is only a broad design rule for the space-time inner code, since it assumes an infinite-length Gaussian-output outer code. A more precise design problem, which remains open, is to find the space-time inner code that achieves minimum error rate for a given finite-length finite-field outer code.

\section{APPENDIX I \\ PROOF OF THEOREM 3}

The intuition behind Theorem 3 is that the multiplexing order $\mu_{\text {eff }}$ is governed by the rank of the effective channel matrix $\mathbf{H}_{\text {eff }}$. Note that $\mathbf{H}_{\text {eff }}=\hat{\mathbf{G}} \mathbf{M}$ cannot have a greater rank than either $\hat{\mathbf{G}}$ or $\mathbf{M}$. Now, it has dimension $2 \mathrm{Nr} \times 2 \mathrm{Nt}$, hence its rank is at most $\min (2 N r, 2 N t)$, or equivalently $2 N \min (t, r)$. Similarly, the $2 N t \times 2 K$ encoding matrix $\mathbf{M}$ has rank at most $2 N \min (t, K / N)$. Combining the two, and noting that $K / N$ is equal to the rate $R$, we see that the maximum rank of $\mathbf{H}_{\text {eff }}$ is $2 N n$, where $n=\min (t, r, R)$. Consequently, if $\nu_{1} \geq \nu_{2} \geq$ $\nu_{2 N r} \geq 0$ are the ordered singular values of $\mathbf{H}_{\mathrm{eff}} \mathbf{H}_{\mathrm{eff}}^{\mathrm{T}}$, then, at most, the first $2 N n$ singular values are nonzero, and the remaining are 0 . Writing out the determinant term in (11) in terms of the singular values $\left\{\nu_{i}\right\}$, we get

$$
\operatorname{det}\left(\mathbf{I}_{2 N r}+\alpha \frac{S}{t} \mathbf{H}_{\mathrm{eff}} \mathbf{H}_{\mathrm{eff}}^{\mathrm{T}}\right)=S^{2 N n} \prod_{i=1}^{2 N n}\left[\frac{\alpha}{t} \nu_{i}+\frac{1}{S}\right] .
$$

Taking logarithm and dividing by $2 N$, we see that the available capacity (11) is given by

$$
J(S, H)=n \log S+\frac{1}{2 N} \log \prod_{i=1}^{2 N n}\left[\frac{\alpha}{t} \nu_{i}+\frac{1}{S}\right] .
$$

By definition, the outage capacity is $D\left(S, p_{\mathrm{o}}\right)=\sup \left\{R_{\mathrm{b}}\right.$ : $\left.\operatorname{Pr}\left[J(S, \mathbf{H})<R_{\mathrm{b}}\right]<p_{\mathrm{o}}\right\}$. Substituting for $J(S, \mathbf{H})$ from (A2), and using the fact that the log function is monotonic and can be moved out of the $\sup \{\cdot\}$, we get

$$
D\left(S, p_{\mathrm{o}}\right)=n \log S+\frac{1}{2 N} \log Y\left(S, p_{\mathrm{o}}\right)
$$

where

$$
Y\left(S, p_{\mathrm{o}}\right)=\sup \left\{y: \operatorname{Pr}\left[\prod_{i=1}^{2 N n}\left(\frac{\alpha}{t} \nu_{i}+\frac{1}{S}\right)<y\right]<p_{\mathrm{o}}\right\} .
$$

Substituting (A3) in (15), we see that the multiplexing order is given by

$$
\mu_{\mathrm{eff}}=n+\frac{1}{2 N} \lim _{S \rightarrow \infty} \frac{\log Y\left(S, p_{\mathrm{o}}\right)}{\log S} .
$$

Thus, one can obtain $\mu_{\mathrm{eff}}$ by evaluating the second limit, involving $Y\left(S, p_{\mathrm{o}}\right)$.

To evaluate the limit, we define the random variable $f\left(S, \mathbf{H}_{\text {eff }}\right)=\prod_{i=1}^{2 N n}\left((\alpha / t) \nu_{i}+(1 / S)\right)$ and its left-continuous distribution function $g(S, y)=\operatorname{Pr}\left[f\left(S, \mathbf{H}_{\text {eff }}\right)<y\right]$. Then, (A4) becomes

$$
Y\left(S, p_{\mathrm{o}}\right)=\sup \left\{y: g(S, y)<p_{o}\right\}
$$

Using (A6), we now prove two lemmas, proving that $Y\left(S, p_{\mathrm{o}}\right)$ is finite and nonzero.

Lemma 1: For all $p_{\mathrm{o}}<1, Y\left(S, p_{\mathrm{o}}\right) \leq Y\left(1, p_{\mathrm{o}}\right)<\infty$ for all SNR $S>1$.

Proof: Note that $S>1 \Rightarrow f\left(S, \mathbf{H}_{\text {eff }}\right)<f\left(1, \mathbf{H}_{\text {eff }}\right) \Rightarrow$ $g(S, y) \geq g(1, y)$ for all $y \Rightarrow Y\left(S, p_{\mathrm{o}}\right) \leq Y\left(1, p_{\mathrm{o}}\right)$. It remains to show that $Y\left(1, p_{\mathrm{o}}\right)<\infty$. The random variable $f\left(1, \mathbf{H}_{\mathrm{eff}}\right)$ is a real-valued transformation of the random elements of $\mathbf{H}$, and is therefore a well-behaved random variable with no point masses at infinity. For all well-behaved random variables, the following property holds [19]

$$
\lim _{y \rightarrow \infty} g(1, y)=\lim _{y \rightarrow \infty} \operatorname{Pr}\left[f\left(1, \mathbf{H}_{\mathrm{eff}}\right)<y\right]=1 .
$$

By definition of limit, $g(1, y)$ approaches its limiting value 1 arbitrarily closely as $y$ increases. Hence, for all $p_{\mathrm{o}}<1$, there is a $Y_{\mathrm{o}}<\infty$ such that $g(1, y)>p_{\mathrm{o}}$ for all $y>Y_{\mathrm{o}}$. From (A6), this implies $Y\left(1, p_{\mathrm{o}}\right) \leq Y_{\mathrm{o}}<\infty$, which proves the lemma.

From Lemma 1 , it is clear that $Y\left(S, p_{\mathrm{o}}\right)$ is bounded as $S$ approaches infinity. Hence, the limit involving $Y\left(S, p_{\mathrm{o}}\right)$ in (A5) is at most 0 , proving that $\mu_{\mathrm{eff}} \leq n$, as claimed in Theorem 3 . We now proceed to prove that $\mu_{\text {eff }}=n$ if $\mathrm{H}_{\text {eff }}$ is full rank with probability 1 .

Lemma 2: If $\mathbf{H}_{\text {eff }}$ is full rank with probability 1, for all $p_{\mathrm{o}}>0$, there is a $y_{\mathrm{o}}>0$ such that $Y\left(S, p_{\mathrm{o}}\right) \geq y_{\mathrm{o}}$ for all SNR $S$.

Proof: It is easy to see that the following inequality holds:

$$
g(S, y)=\operatorname{Pr}\left[\prod_{i=1}^{2 N n}\left(\frac{\alpha}{t} \nu_{i}+\frac{1}{S}\right)<y\right] \leq \operatorname{Pr}\left[\prod_{i=1}^{2 N n} \frac{\alpha}{t} \nu_{i}<y\right]
$$

The right-hand side is obtained by setting $S=\infty$ in the definition of $g(S, y)$, hence, we call it $g(\infty, y)$. As $y \rightarrow 0$, its limit is given by

$$
\lim _{y \rightarrow 0} \operatorname{Pr}\left[\prod_{i=1}^{2 N n} \frac{\alpha}{t} \nu_{i}<y\right]=\operatorname{Pr}\left[\prod_{i=1}^{2 N n} \frac{\alpha}{t} \nu_{i} \leq 0\right]
$$

The right-hand side of (A9) is the probability that at least one of the singular values $\left\{\nu_{i}\right\}$ is 0 , or that $\mathbf{H}_{\text {eff }}$ is not full rank, which is 0 by hypothesis. This implies that as $y \rightarrow 0$, $g(\infty, y) \rightarrow 0$. Hence, for any $p_{\mathrm{o}}>0$, there is a $y_{\mathrm{o}}>0$ such that $g(\infty, y)<p_{\mathrm{o}}$ for all $y<y_{\mathrm{o}}$. Using $g(S, y) \leq g(\infty, y)$ from (A8), we conclude $g(S, y)<p_{\mathrm{o}}$ for all $y<y_{\mathrm{o}}$, which from (A6) implies $Y\left(S, p_{\mathrm{o}}\right) \geq y_{\mathrm{o}}$. This proves the lemma.

If $\mathbf{H}_{\text {eff }}$ is full rank with probability 1 , Lemma 2 gives the lower bound $Y\left(S, p_{\mathrm{o}}\right) \geq y_{\mathrm{o}} \geq 0$. Substituting in (A6), the limit involving $Y\left(S, p_{\mathrm{o}}\right)$ is at least 0 , implying $\mu_{\mathrm{eff}} \geq n$. From Lemma 1, we know $\mu_{\mathrm{eff}} \leq n$. Thus, we conclude that $\mu_{\mathrm{eff}}=n$ if $\mathbf{H}_{\text {eff }}$ is full rank with probability 1, as claimed in Theorem 3 . 


\section{APPENDIX II \\ PROOF OF THEOREM 4}

We seek conditions to ensure $\mathbf{H}_{\text {eff }}=\hat{\mathbf{G} M}$ is full rank with probability 1 . Note that $R \leq \min (t, r) \Rightarrow K=N R \leq$ $N \min (t, r) \Rightarrow \hat{\mathbf{G}} \mathbf{M}$ and $\mathbf{M}$ both have at least as many rows as columns. Consequently, $\hat{\mathbf{G M}}$ and $\mathbf{M}$ are full rank if and only if their columns are linearly dependent. Now, $\mathbf{M}$ is not full rank $\Rightarrow$ there is some $\mathbf{u}_{\mathbf{M}} \neq \mathbf{0}$ such that $\mathbf{M} \mathbf{u}_{\mathbf{M}}=\mathbf{0} \Rightarrow \hat{\mathbf{G M}} \mathbf{M u} \mathbf{u}_{\mathbf{M}}=$ $\mathbf{0} \Rightarrow \hat{\mathbf{G} M}$ is also not full rank for all $\hat{\mathbf{G}}$. Thus, to ensure $\hat{\mathbf{G M}}$ is full rank with probability 1 , it is necessary that $\mathbf{M}$ also has full rank, proving the first part of Theorem 4.

We now proceed to prove the sufficient conditions 1 and 2 , assuming $\mathbf{M}$ is full rank. Under this assumption, the nonzero column span of $\mathbf{M}$, namely $\mathcal{X}=\{\mathbf{x}: \hat{\mathbf{x}}=\mathbf{M} \hat{\mathbf{u}}$ for some $\hat{\mathbf{u}} \neq \mathbf{0}\}$ does not contain the zero vector. Now, $\hat{\mathbf{G M}}$ is not full rank $\Leftrightarrow$ there is some $\mathbf{x} \in \mathcal{X}$ such that $\hat{\mathbf{G}} \hat{\mathbf{x}}=\mathbf{0}$, or equivalently $\mathbf{G x}=\mathbf{0}$. In other words, $\hat{\mathbf{G M}}$ is not full rank if and only if the random Rayleigh fading channel matrix $\mathbf{H}$ belongs to the bad channel set

$$
\mathcal{B}=\{\mathbf{H}: \mathbf{G} \mathbf{x}=\mathbf{0} \text { for some } \mathbf{x} \in \mathcal{X}\}
$$

To prove $\hat{\mathbf{G M}}$ is full rank with probability 1 , we need to prove $\operatorname{Pr}[\mathbf{H} \in \mathcal{B}]=0$.

Proof of 1: First, consider the case $r \geq t$. Clearly, since $\mathcal{X}$ does not contain the zero vector, $\mathbf{H} \in \mathcal{B} \Rightarrow \mathbf{G x}=\mathbf{0}$ for some $\mathbf{x} \neq 0 \Leftrightarrow \mathbf{G}$ has linearly dependent columns $\Leftrightarrow \mathbf{H}$ has linearly dependent columns using the block diagonal structure of $\mathbf{G}$. Since $r \geq t, \mathbf{H}$ has linearly dependent columns $\Leftrightarrow \mathbf{H}$ is not full rank. Thus, $\mathbf{H} \in \mathcal{B} \Rightarrow \mathbf{H}$ is not full rank, giving the bound $\operatorname{Pr}[\mathbf{H} \in \mathcal{B}] \leq \operatorname{Pr}[\mathbf{H}$ does not have full rank $]$. For Rayleigh fading channels, the latter probability is known [11] to be 0 , implying $\operatorname{Pr}[\mathbf{H} \in \mathcal{B}]=0$, or equivalently that $\hat{\mathbf{G M}}$ is full rank with probability 1 , hence $\mu_{\mathrm{eff}}=R$. This proves condition 1.

Proof of 2: When $r<t, \quad \operatorname{Pr}[\mathbf{H}$ has linearly dependent columns $]=1$, so the above method cannot be used to guarantee $\operatorname{Pr}[\mathbf{H} \in \mathcal{B}]=0$. We need to show that $\operatorname{Pr}[\mathbf{H} \in \mathcal{B}]=$ 0 if the assumptions of condition 2 hold. Now, $\mathbf{H} \in \mathcal{B} \Leftrightarrow \mathbf{G} \mathbf{x}=$ $\mathbf{0}$ for some $\mathbf{x} \in \mathcal{X} \Leftrightarrow \mathbf{H x}_{i}=\mathbf{0}$ for all the transmit vectors $\mathbf{x}_{i}$ corresponding to the composite vector $\mathbf{x}$. Let $\mathcal{X}_{i}=\left\{\mathbf{x}_{i}: \mathbf{x} \in\right.$ $\mathcal{X}\}$, and define the local bad channel set

$$
\mathcal{B}_{i}=\left\{\mathbf{H}: \mathbf{H x}_{i}=\mathbf{0} \text { for some } \mathbf{x}_{i} \in \mathcal{X}_{i}, \mathbf{x}_{i} \neq \mathbf{0}\right\} .
$$

Then, $\mathbf{H} \in \mathcal{B} \Rightarrow \mathbf{H} \in \mathcal{B}_{i}$ for some $i=1,2, \ldots, N$. Equivalently, $\mathcal{B} \subseteq \bigcup_{i=1}^{N} \mathcal{B}_{i}$. To prove $\operatorname{Pr}[\mathbf{H} \in \mathcal{B}]=0$, it suffices to prove $\operatorname{Pr}\left[\mathbf{H} \in \mathcal{B}_{i}\right]=0$ for all $i$. By assumption, every $\mathbf{x}_{i} \in \mathcal{X}_{i}$ also belongs to a complex linear space $\mathcal{S}_{i}$ of dimension $l_{i}$, so it can be written as the column span of a $t \times l_{i}$ complex matrix $\mathbf{B}_{i}$ with orthonormal columns, i.e., $\mathbf{x}_{i}=\mathbf{B}_{i} \mathbf{u}_{i}$ for some nonzero $\mathbf{u}_{i}$. Substituting in (B2), the local bad set now is

$$
\mathcal{B}_{i}=\left\{\mathbf{H}: \mathbf{H B}_{i} \mathbf{u}_{i}=\mathbf{0} \text { for some } \mathbf{u}_{i} \neq 0\right\} .
$$

Define the transformed random matrix $\mathbf{H}_{i}^{\prime}=\mathbf{H B}_{i}$. Then, $\mathbf{H} \in \mathcal{B}_{i} \Leftrightarrow \mathbf{H B}_{i} \mathbf{u}_{i}=\mathbf{H}_{i}^{\prime} \mathbf{u}_{i}=\mathbf{0}$ for some $\mathbf{u}_{i} \neq \mathbf{0} \Leftrightarrow \mathbf{H}_{i}^{\prime}$ has linearly dependent columns. Now, it is easy to show [11] that $\mathbf{H}_{i}^{\prime}=\mathbf{H B}_{i}$ is a Rayleigh fading matrix of dimension $r \times l_{i}$. Further, since $l_{i} \leq r$ by assumption, $\operatorname{Pr}\left[\mathbf{H}_{i}^{\prime}\right.$ has linearly dependent columns $]=\operatorname{Pr}\left[\mathbf{H}_{i}^{\prime}\right.$ is not full rank $]=0$. Thus, $\operatorname{Pr}\left[\mathbf{H} \in \mathcal{B}_{i}\right]=0$. The same argument applies for all $i=1,2$, $\ldots, N$, hence from $\mathcal{B} \subseteq \bigcup_{i=1}^{N} \mathcal{B}_{i}$, we see that $\operatorname{Pr}[\mathbf{H} \in \mathcal{B}=0]$. This completes the proof of condition 2 .

\section{REFERENCES}

[1] L. Zheng and D. N. C. Tse, "Diversity and multiplexing: A fundamental trade-off in multiple antenna channels," IEEE Trans. Inf. Theory, vol. 49, no. 5, pp. 1073-1096, May 2003.

[2] V. Tarokh, N. Seshadri, and A. Calderbank, "Space-time codes for high data rate wireless communication: Performance criterion and code construction," IEEE Trans. Inf. Theory, vol. 44, no. 2, pp. 744-765, Mar. 1998.

[3] S. M. Alamouti, "A simple transmitter diversity scheme for wireless communications," IEEE J. Sel. Areas Commun., vol. 16, no. 8, pp. 1451-1458, Oct. 1998.

[4] V. Tarokh, H. Jafarkhani, and A. Calderbank, "Space-time block codes from orthogonal designs," IEEE Trans. Inf. Theory, vol. 45, no. 5, pp. 1456-1467, Jul. 1999.

[5] X. Ma and G. B. Giannakis, "Full-diversity full-rate complex-field space-time coding," IEEE Trans. Signal Process., vol. 51, no. 11, pp. 2917-2930, Nov. 2003.

[6] B. M. Hochward and S. T. Brink, "Achieving near-capacity on a multipleantenna channel," IEEE Trans. Commun., vol. 51, no. 3, pp. 389-399, Mar. 2003.

[7] V. Gulati and K. R. Narayanan, "Concatenated space-time codes for quasi-static fading channels: Constrained capacity and code design," in IEEE Global Telecommunications Conf. (GLOBECOM), Taipei, Taiwan, Nov. 2002, vol. 2, pp. 1202-1206.

[8] G. J. Foschini and M. J. Gans, "On the limits of wireless communications in a fading environment when using multiple antennas," Wireless Pers. Commun., vol. 6, no. 3, pp. 311-335, Mar. 1998.

[9] R. Heath and A. Paulraj, "Linear dispersion codes for MIMO systems based on frame theory," IEEE Trans. Signal Process., vol. 50, no. 10, pp. 2429-2441, Oct. 2002.

[10] B. Hassibi and B. M. Hochwald, "High-rate codes that are linear in space and time," IEEE Trans. Inf. Theory, vol. 48, no. 7, pp. 1804-1824, Jul. 2002.

[11] I. E. Telatar, "Capacity of multi-antenna Gaussian channels," Eur. Trans. Telecommun., vol. 10, no. 6, pp. 585-595, Nov./Dec. 1999.

[12] S. Sandhu and A. Paulraj, "Unified design of linear space-time block codes," in Proc. Global Telecommunications Conf. (GLOBECOM), San Antonio, TX, Nov. 2001, vol. 2, pp. 1073-1077.

[13] B. Papadias and G. J. Foschini, "On the capacity of certain space-time coding schemes," Eurasip J. Appl. Signal Process., vol. 2002, no. 5, pp. 447-458, May 2002.

[14] S. Sandhu and A. Paulraj, "Space-time block coding: A capacity perspective," IEEE Commun. Lett., vol. 4, no. 12, pp. 384-386, Dec. 2000.

[15] G. Golden, G. Foschini, R. Venezuela, and P. Wolniansky, "Detection algorithm and initial laboratory results using V-BLAST space-time communication architecture," IEEE Electron. Lett., vol. 35, no. 1, pp. 14-16, Jan. 1999.

[16] B. Varadrajan and J. R. Barry, "The rate-diversity trade-off for linear space-time codes," in Proc. IEEE Vehicular Technology Conf., Vancouver, BC, Canada, Sep. 2002, vol. 1, pp. 67-71.

[17] B. Varadarajan, "The design of linear space-time codes for quasi-static flat-fading channels," Ph.D. dissertation, Dept. Elect. Comput. Eng., Georgia Inst. Technol., Atlanta, 2004.

[18] V. Tarokh, A. Naguib, N. Seshadri, and A. Calderbank, "Combined array processing and space-time coding," IEEE Trans. Inf. Theory, vol. 45, no. 4, pp. 1121-1128, May 1999.

[19] A. Papoulis, Probability, Random Variables and Stochastic Processes. New York: McGraw-Hill, 1991. 